\title{
New Insights on the Importance of the Extent of Vascular Invasion in Encapsulated Angio-invasive Follicular Thyroid Carcinoma
}

\author{
Danielli Matsuura, MD, and Ian Ganly, MD, MS, PhD \\ Department of Surgery, Head and Neck Service, Memorial Sloan Kettering Cancer Center, New York, NY
}

The significance of the extent of vascular invasion (VI) in thyroid cancer has been thoroughly investigated and debated in the literature, and yet there is always more to discover, as described in the article "Encapsulated Angioinvasive Follicular Thyroid Carcinoma: Prognostic Impact of the Extent of Vascular Invasion" by Yamazaki et al. ${ }^{1}$ In this study, Yamazaki et al. have revisited the significance of the number of foci of VI on recurrence and survival in patients with thyroid cancer. Notably, only patients with encapsulated angio-invasive follicular thyroid carcinoma (FTC) were included in this study with a relatively large final cohort of 264 patients. Following receiver operating characteristic (ROC) curve analysis, a new optimal cutoff-VI foci of 2-was identified for both cause-specific survival (CSS) [area under the curve (AUC) $=0.60$ (sensitivity, 1.00; specificity, 0.40)] and disease-free survival (DFS) $[\mathrm{AUC}=0.67$ (sensitivity, 0.90; specificity, 0.45)]. Moreover, patients with 1 focus of VI had statistically significant superior CSS and DFS rates compared with patients who had 2 or more foci of VI. The 10-year CSS rates were $100 \%$ and $94.5 \%$, respectively, with a $p$ value of 0.042. The 10-year DFS rates showed even more striking differences $(94.9 \%$ and $77.9 \% ; p<0.001)$. Surprisingly, when comparing the usual classification of 1-3 foci versus 4 or more foci, there was no statistically significant difference for CSS $(p=0.597)$ or DFS $(p=0.311)$. Age

(C) Society of Surgical Oncology 2022

First Received: 17 January 2022

Accepted: 20 January 2022;

Published Online: 6 February 2022

I. Ganly, MD, MS, PhD

e-mail: ganlyi@mskcc.org $\geq 55$ years along with 2 or more foci of VI were reported as significant negative prognostic factors for DFS in a multivariate analysis.

The extent of VI has been a controversial topic in thyroid cancer since it was first described by Graham in 1924 . $^{2}$ The very definition of VI in thyroid cancer has evolved and changed without ever reaching an absolute consensus regarding the criteria to characterize VI. The impact of the mere presence of VI on survival outcomes has been also debated. Sugino et al. showed that VI did not increase the risk for distant metastases. ${ }^{3}$ In contrast, Mete and $\mathrm{Asa}^{4}$ highlighted the presence of VI as a valuable predictor of developing distant metastasis, especially in well-differentiated thyroid cancer, and this has been corroborated by several other studies. ${ }^{5-10}$ The relevance of the number of foci of VI also has been questioned. Wreesmann et al. ${ }^{7}$ did not find a significant difference comparing $<4$ and $\geq 4$ foci of VI in well-differentiated papillary thyroid carcinomas (PTCs), granted that PTCs were included. However, in a cohort of 276 patients with low-grade, encapsulated, follicular, cell-derived, thyroid carcinomas, $\mathrm{Xu}$ et al. ${ }^{11}$ reported that extensive VI ( $\geq 4$ foci) was an independent prognostic predictor of recurrence. Ghossein et al. ${ }^{5}$ also demonstrated that $\geq 4$ foci of VI was the most important predictor of recurrence in patients with encapsulated Hürthle cell carcinoma (HCC). Recurrence-free survival rates were significantly higher for patients with $<4$ foci compared with $\geq 4$ foci (100\% vs. $20 \%$, respectively; $p<0.001)^{5}$

Notably, the existing literature on this matter diverges not only on the results but also the inclusion criteria and the classification of tumors. As noted by Yamazaki et al. ${ }^{1}$ most of the previous studies included different histological types of thyroid carcinomas to evaluate VI. Furthermore, over the years the nomenclature and criteria to classify tumors also have changed, making it difficult to compare prior 
studies. For instance, FTC encompassed HCC tumors until 2017 when HCC was finally classified as an independent entity. Using the extent of VI, FTC was traditionally classified into minimally invasive and widely invasive. ${ }^{12-14}$ In 2014, the Armed Forces Institute of Pathology subdivided minimally invasive tumors into 3 subcategories: tumors with only capsular invasion; tumors with limited VI ( $<4$ foci); and tumors with extensive vascular VI $(>4$ foci). Lastly, in 2017, the World Health Organization Classification of Endocrine Tumors ${ }^{15}$ grouped FTC into 3 categories: minimally invasive (capsular invasion only); encapsulated angio-invasive (presence of angioinvasion, regardless of the number of foci); and widely invasive. These modifications underline how much has changed in the classification of these tumors throughout the years.

Another limitation of the aforementioned studies is the very nature of FTCs and HCCs, rare types of thyroid cancers. The cohorts are limited to few patients as are the events of interest, such as death or recurrence, thereby impacting the study power and generalizability. However, Yamazaki et al. ${ }^{1}$ presents one of the largest series studied in the literature on this topic including only encapsulated angio-invasive FTC. Another highlight of this study is the comprehensive pathological review that provides detailed information and allows reproducibility.

The study by Yamazaki et al. ${ }^{1}$ not only emphasizes the importance of VI on survival outcomes in patients with encapsulated angio-invasive FTC but also brings awareness that patients with 2 or more foci, especially patients older than aged 55 years, might present poorer clinical outcomes. This study will pave the way for new studies that could validate these findings in different target populations, leading to possible impactful changes in the current practice regarding extent of surgery, adjuvant treatments, and follow-up of these patients. Lastly, and maybe most importantly, it reminds us to always question the established cutoffs and truths in order to advance science and patient care.

\section{DECLARATIONS}

DISCLOSURES The authors declare no conflicts of interest.

\section{REFERENCES}

1. Yamazaki H KR, Sugino K, Matsuzu K, Masaki C, Akaishi J, Hames KY, Tomoda C, Suzuki A, Ohkuwa K, Kitagawa W,
Nagahama M, Rino Y, Ito K. Encapsulated angio-invasive follicular thyroid carcinoma: prognostic impact of the extent of vascular invasion. Ann Surg Oncol. 2022. https://doi.org/10.1245/ s10434-022-11401-x.

2. Graham A. Malignant epithelial tumors of the thyroid with special reference to invasion of blood vessels. Surg Gynecol Obstet. 1924;39:781-90.

3. Sugino K, Kameyama K, Ito K, et al. Outcomes and prognostic factors of 251 patients with minimally invasive follicular thyroid carcinoma. Thyroid. 2012;22(8):798-804.

4. Mete O, Asa SL. Pathological definition and clinical significance of vascular invasion in thyroid carcinomas of follicular epithelial derivation. Mod Pathol. 2011;24(12):1545-52.

5. Ghossein RA, Hiltzik DH, Carlson DL, et al. Prognostic factors of recurrence in encapsulated Hurthle cell carcinoma of the thyroid gland: a clinicopathologic study of 50 cases. Cancer. 2006;106(8):1669-76.

6. Lang W, Choritz H, Hundeshagen H. Risk factors in follicular thyroid carcinomas. A retrospective follow-up study covering a 14-year period with emphasis on morphological findings. Am J Surg Pathol. 1986;10(4):246-55.

7. Wreesmann VB, Nixon IJ, Rivera M, et al. Prognostic value of vascular invasion in well-differentiated papillary thyroid carcinoma. Thyroid. 2015;25(5):503-8.

8. Ortiz S, Rodríguez JM, Soria T, et al. Extrathyroid spread in papillary carcinoma of the thyroid: clinicopathological and prognostic study. Otolaryngol Head Neck Surg. 2001;124(3):261-5.

9. Collini P, Sampietro G, Pilotti S. Extensive vascular invasion is a marker of risk of relapse in encapsulated non-Hürthle cell follicular carcinoma of the thyroid gland: a clinicopathological study of 18 consecutive cases from a single institution with a 11-year median follow-up. Histopathology. 2004;44(1):35-9.

10. Stojadinovic A, Ghossein RA, Hoos A, et al. Hürthle cell carcinoma: a critical histopathologic appraisal. J Clin Oncol. 2001;19(10):2616-25.

11. Xu B, Wang L, Tuttle RM, Ganly I, Ghossein R. Prognostic impact of extent of vascular invasion in low-grade encapsulated follicular cell-derived thyroid carcinomas: a clinicopathologic study of 276 cases. Hum Pathol. 2015;46(12):1789-98.

12. van Heerden JA, Hay ID, Goellner JR, et al. Follicular thyroid carcinoma with capsular invasion alone: a nonthreatening malignancy. Surgery. 1992;112(6):1130-6; discussion 1136-8.

13. Ito $Y$, Hirokawa M, Higashiyama T, et al. Prognosis and prognostic factors of follicular carcinoma in Japan: importance of postoperative pathological examination. World $J$ Surg. 2007;31(7):1417-24.

14. Kim HJ, Sung JY, Oh YL, et al. Association of vascular invasion with increased mortality in patients with minimally invasive follicular thyroid carcinoma but not widely invasive follicular thyroid carcinoma. Head Neck. 2014;36(12):1695-700.

15. WHO Classification of Tumours of Endocrine Organs. Vol 10. 4th edn. Lyon, France: IARC Publications; 2017.

Publisher's Note Springer Nature remains neutral with regard to jurisdictional claims in published maps and institutional affiliations. 